Reprod. Nutr. Dévelop., 1987, 27 (1 B), 281-282.

\title{
Digestibilité et ingestibilité des fourrages verts chez le mouton : effets respectifs du niveau d'alimentation et de l'âge ou du poids des animaux
}

C. DEMARQUILLY, J. ANDRIEU

Laboratoire des Aliments,

I.N.R.A. Theix 63122 Ceyrat, France.

Summary. The organic matter digestibility of fresh grass offered ad libitum to sheep was only 0.016 unit lower than that offered at maintenance level. Digestibility and intake (in $\left.\mathrm{g} / \mathrm{kg} \mathrm{W}^{0.75}\right)$ were independent of sheep age after 10 months.

La digestibilité et l'ingestibilité des fourrages verts données dans les "Tableaux de la valeur alimentaire des fourrages " (Demarquilly et Weiss, 1970) ou dans les "Tableaux de la valeur nutritive des aliments " (Demarquilly et al., 1978) ont été obtenues sur des lots de 6 moutons alimentés à volonté. Dès 1962, nous avons en effet choisi de déterminer la digestibilité sur des moutons alimentés à volonté et non en quantité limitée, voisine de l'entretien des animaux. Deux raisons avaient motivé ce choix : 1) nous pouvions dans une même mesure déterminer à la fois la digestibilité et l'ingestibilité du fourrage et 2) les résultats publiés montraient une influence faible du niveau d'alimentation des moutons sur la digestibilité des fourrages verts (Raymond et al., 1959 ; Andersen et al., 1959). Ce dernier point devait cependant être vérifié, de même que la validité de l'exposant du poids $(0,75)$ pour exprimer les quantités ingérées.

\section{Matériel et méthodes.}

Essai 1. Deux lots de 6 moutons de 18 mois appariés et maintenus en cages à digestibilité ont reçu du fourrage vert de dactyle à volonté $(10 \%$ refus, lot 1$)$ ou en quantité limitée (40 g MS/kg P0,75, lot 2) au cours des $1^{\mathrm{er}}, 2^{\mathrm{e}}$ et $3^{\mathrm{e}}$ cycles. Pour chaque cycle, après une période d'adaptation de 8 jours, la digestibilité a été mesurée en continu, chaque période ( 7 au $1^{\mathrm{e}}, 4$ au $2^{\mathrm{e}}$ et 4 au $3^{\mathrm{e}}$ cycle) de mesure de 6 jours étant séparée de la suivante par un seul jour, le samedi.

Essai 2. Deux lots de 12 moutons mâles castrés de race Texel $(4$ de 10 mois, 4 de 22 mois et 4 de 3 ans ou plus) appariés 2 à 2 sur leur âge, leur poids et leur capacité d'ingestion et maintenus en cage à digestibilité ont reçu un ray-grass feuillu (semis de l'année). Dans une première période le lot 1 a reçu le fourrage à volonté (10\% de refuis) et le lot 2 en quantité limitée égale à $60 \%$ de la quantité ingérée par le lot 1. Dans une deuxième période les lots ont été inversés. Chaque période a duré 20 jours, la digestibilité et l'ingestibilité étant déterminées pendant les 9 derniers jours.

Dans les 2 essais, le fourrage était fauché chaque matin, haché dans un hache-paille en brins de 3 à $5 \mathrm{~cm}$ pour limiter le tri et distribué en 2 fois.

\section{Résultats et discussion.}

- Influence du niveau d'alimentation sur la digestibilité. - Les animaux des 2 essais, alimentés à volonté, ont consommé en moyenne $60,8 \mathrm{~g}$ de MS $/ \mathrm{kg} \mathrm{P} \mathrm{P}^{0,75}$ (de 75,9 à $42,8 \mathrm{~g}$ suivant les 17 échantillons) et ceux en quantité limitée $41,8 \mathrm{~g}$ (de 39,6 à $46,6 \mathrm{~g}$ ) soit $30 \%$ de moins. La digestibilité de la matière organique (dMO) 
correspondante a été respectivement de 0,684 (de 0,590 à 0,791 ) et de 0,700 (de $0,606$ à 0,812$)$, soit une différence de 0,016 unité $(2,2 \%)$, significative $(P<0,001$ dans la comparaison des 17 couples), mais faible et indépendante du stage de végétation. Si nous la rapportons au niveau d'alimentation (NA $=1$ à l'entretien), qui est passé en moyenne de 1,14 à 1,63, la diminution de la dMO ( $\Delta \mathrm{dMO}$ ) est donc de 0,033 unité pour une augmentation de $1 \mathrm{du}$ NA. En réalité pour un même échantillon $\Delta$ dMO a été indépendante de la différence de $N A(\Delta N A)(r=0,057$ entre $\Delta$ dMO et $\Delta$ NA pour $n=17$ ). Elle semble donc résulter surtout du mode de distribution (ad libitum ou quantité limitée). Ces résultats confirment donc les données bibliographiques antérieures. L'augmentation avec le niveau d'alimentation de la teneur en matière organique non digestible est expliquée pour $5 \%$ par celle des matières azotées non digestibles et pour $48 \%$ par celle de la cellulose brute non digestible.

- Influence de l'âge et du poids des moutons sur la digestibilité. - Dans l'essai 2, les différences de digestibilité suivant l'âge ou le poids moyen des différents lots de 4 moutons ont toujours été très faibles ( $<0,01$ unité) et non significatives (tabl. 1). Raymond et al. (1954) ont cependant observé une légère augmentation $(0,01$ unité) de la digestibilité entre l'âge de 1 et de 2 ans chez des moutons.

TABL. 1. - Influence de l'âge et du poids des moutons sur la digestibilité (essai 2).

\begin{tabular}{|c|c|c|c|c|c|c|}
\hline \multirow{2}{*}{$\begin{array}{l}\text { Pé- } \\
\text { riode }\end{array}$} & \multicolumn{4}{|c|}{ Moutons } & \multicolumn{2}{|c|}{ Digestibilité } \\
\hline & Nombre & Age & QL (1) & ADL (2) & $\mathrm{QL}(1)$ & ADL (2) \\
\hline P1 & $\begin{array}{l}2 \times 4 \\
2 \times 4 \\
2 \times 4\end{array}$ & $\begin{array}{l}3 \text { à } 5 \text { ans } \\
22 \text { mois } \\
10 \text { mois }\end{array}$ & $\begin{array}{l}73,0 \mathrm{~kg} \\
49,5 \mathrm{~kg} \\
32,2 \mathrm{~kg}\end{array}$ & $\begin{array}{l}78,6 \mathrm{~kg} \\
55,6 \mathrm{~kg} \\
37,0 \mathrm{~kg}\end{array}$ & $\begin{array}{l}0,813 \pm 0,007 \\
0,811 \pm 0,003 \\
0,813 \pm 0,003\end{array}$ & $\begin{array}{l}0,795 \pm 0,016 \\
0,786 \pm 0,018 \\
0,793 \pm 0,020\end{array}$ \\
\hline $\mathrm{P} 2$ & $\begin{array}{l}2 \times 4 \\
2 \times 4 \\
2 \times 4\end{array}$ & $\begin{array}{l}3 \text { à } 5 \text { ans } \\
22 \text { mois } \\
10 \text { mois }\end{array}$ & $\begin{array}{l}75,0 \mathrm{~kg} \\
54,9 \mathrm{~kg} \\
35,9 \mathrm{~kg}\end{array}$ & $\begin{array}{l}80,1 \mathrm{~kg} \\
56,6 \mathrm{~kg} \\
34,2 \mathrm{~kg}\end{array}$ & $\begin{array}{l}0,799 \pm 0,008 \\
0,804 \pm 0,005 \\
0,809 \pm 0,014\end{array}$ & $\begin{array}{l}0,780 \pm 0,016 \\
0,788 \pm 0,013 \\
0,790 \pm 0,007\end{array}$ \\
\hline
\end{tabular}

(1) $\mathrm{QL}=$ quantité limitée ; (2) $\mathrm{ADL}=$ quantité ad libitum.

Mode d'expression des quantités ingérées. - Les quantités de matière sèche ingérées (Ql) par les 12 moutons alimentés à volonté, en période P1 de l'essai 2, ont été liées aux poids ( $P$ en $\mathrm{kg}$ variant de 30,5 à $85,0 \mathrm{~kg}$ ) des moutons par les relations suivantes:

$\begin{array}{llll}\text { Ol en g/jour } & 297,9+22,25 & \mathrm{P} \pm 129 & \mathrm{r}=0,959^{* * *} \\ \text { Ql en } \mathrm{g} / 100 \mathrm{~kg} \text { poids vif } & =3376-10,026 \mathrm{P} \pm 226 & \mathrm{r}=0,658^{*} \\ \text { Ql en } \mathrm{g} / \mathrm{kg} \mathrm{P0,75} & =71,8+0,0714 \mathrm{P} \pm 5,99 & \mathrm{r}=0,229^{\mathrm{Ns}} \text {. }\end{array}$

Seule la quantité ingérée en $\mathrm{g} / \mathrm{kg} \mathrm{P}^{0,75}$ est statistiquement indépendante du poids des animaux. Elle peut donc servir à caractériser l'ingestibilité du fourrage. L'exposant du poids annulant totalement l'influence du poids est de 0,80 et n'est pas significativement différent de 0,75.

Andersen P. E., Reid J. T., Anderson M. J., Stoud J. W., 1959. J. anim. Sci., 18, 1299-1307. Demarquilly C., Weiss Ph., 1970. Etude SEI $n^{\circ} 42$.

Demarquilly C., Andrieu J., Sauvant D., 1978. In L'alimentation des ruminants. Ed. INRA Publications, 78000 Versailles.

Raymond W. F., Harris C. E., Kemp C. D., 1954. J. brit. Grassl. Soc., 9, 209-220.

Raymond W. F., Minson D. J., Harris C. E., 1959. J. brit. Grass/. Soc., 14, 75-77. 